\title{
Producción de nanofibras poliméricas mediante el proceso de electrospinning y su uso potencial
}

\section{Production of polymeric nanofibers by electrospinning process and its potential use}

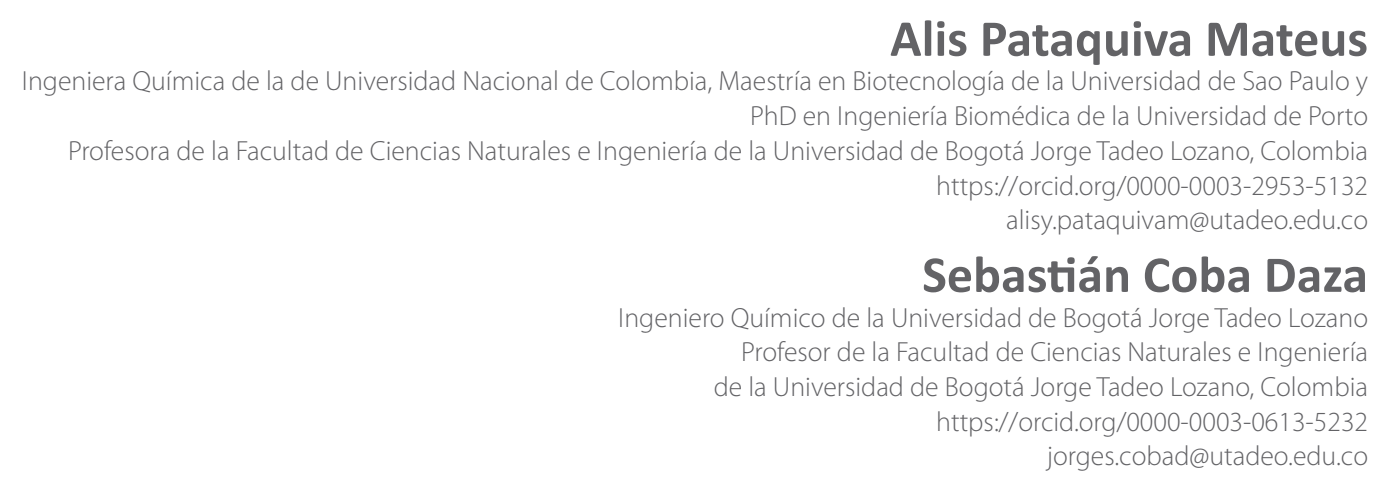

Fecha de recepción: 14 de noviembre del 2017

Fecha de aceptación: 10 de septiembre del 2018

Sugerencia de citacion: Pataquiva Mateus, A. y Coba Daza, S. (2018) Producción de nanofibras poliméricas mediante el proceso de electrospinning y su uso potencial. Mutis 8(1) 17-33, doi: http://dx.doi.org/10.21789/22561498.1375

Editor: Gerard, Olivar-Tost

\section{RESUMEN}

En este artículo de revisión se presentan las diferentes formas de fabricación de nanofibras de polímeros haciendo énfasis tanto en la técnica de electrospinning, la cual es un proceso sencillo, versátil y adecuado para la fabricación de materiales unidimensionales (1D), como en los parámetros que se deben considerar al utilizar este método; además, se señala la importancia de los parámetros de procesamiento (voltaje, distancia y flujo), de solución (concentración, viscosidad y tensión superficial) y ambientales (temperatura y humedad). Finalmente, se mencionan las distintas aplicaciones que involucran nanofibras en campos de acción como energía, ingeniería de tejidos, medio ambiente y alimentos.

Palabras clave: fibras, electrospinning, aplicaciones, nanotecnología.

\section{ABSTRACT}

This review article presents different ways to synthesize polymer nanowires emphasizing the electrospinning technique, which is a simple, versatile, and suitable process for the production of unidimensional structures (1D), and the parameters that should be considered when using this method; besides, it accents the importance of processing parameters (voltage, distance, and flow), solution parameters (concentration, viscosity, and superficial 
tension), and environmental parameters (temperature and humidity). Finally, it shows the different applications of nanowires in fields such as energy, tissue engineering, environment, and food.

Keywords: nanofibers, electrospinning, applications, nanotechnology.

\section{INTRODUCCIÓN}

El uso creciente de materiales unidimensionales (1D) como nanofibras, nanohojas, nanotubos, nanocables y nanocintas ha aumentado significativamente en los últimos años, debido a sus propiedades y a su gran potencial de aplicabilidad (Alivisatos et al., 1998; Ozin, 1992; Schulz, 2000; Wang, Shen, Yao y Park, 2009; Wernsdorfer y Sessoli, 1999). Las técnicas para la creación de dichos materiales son muy variadas e incluyen la litografía, la vía hidrotérmica, así como la deposición química en fase vapor (Wang, 2000).

Cuando las estructuras pasan de tener un tamaño micrométrico a submicrométrico, sus propiedades cambian (Haider, Haider y Kang, 2015). En la Tabla 1 se muestra como estos cambios se ven reflejados en la aparición de características importantes (ZhengMing, Zhang, Kotaki y Ramakrishna, 2003).

Tabla 1. Comparación de propiedades de un material al reducir su tamaño

\begin{tabular}{c|c}
\hline Tamaño micrométrico & Tamaño submicrométrico \\
\hline Baja proporción área/volumen & Alta proporción área/volumen \\
Bajo desempeño de propiedades mecánicas & Alto desempeño de propiedades mecánicas \\
Baja capacidad de funcionalizar la superficie & Alta capacidad de funcionalizar la superficie \\
\hline
\end{tabular}

Fuente: elaboración propia.

La técnica de electrospinning, también llamada electrohilado, hace uso de las fuerzas eléctricas para producir fibras de tamaño micro y nanométrico (Ahn et al., 2006): se realiza una descarga de alto voltaje sobre la solución polimérica, la cual forma un cono llamado cono de Taylor, del cual se desprenden las fibras (Doshi y Reneker, 1995; Taylor, 1969). Diferentes parámetros -como voltaje, caudal, distancia, humedad, concentración del polímero usado, entre otros- influyen en el proceso (Li y Wang, 2013a), por lo que es necesario tener en cuenta cada uno de estos a fin de proporcionar el tamaño de fibra adecuado según los requerimientos.

El aumento de publicaciones ${ }^{1}$ acerca de la técnica de electrospinning para la producción de nanofibras se debe a la facilidad de esta técnica (Haider et al., 2015),

1 Por publicaciones se entiende: artículos científicos, documentos de conferencias, revisiones, artículos de prensa, revisión de conferencias, capítulos de libros, erratas, notas, encuestas cortas, editoriales, libros, cartas, artículos de negocios e informes. al volumen que puede producir, a su fácil escalabilidad (Da Silva Vaz, Vieira Costa y De Morais, 2017) y a la aplicabilidad del producto obtenido (Liang, Hsiao y Chu, 2007). En la Figura 1 se muestra el número de publicaciones en la base de datos Scopus (2017) que incluyen la palabra electrospinning entre 2007 y 2017; se evidencia que esta técnica ha atraído cada vez más la atención de los investigadores.

En este artículo de revisión se presentan las diferentes rutas de fabricación para la producción de nanofibras, y se profundiza en la técnica de electrospinning, haciendo énfasis en el efecto de las variables de operación y en las promisorias aplicaciones de este tipo de material en distintos campos de aplicación.

\section{RUTAS DE FABRICACIÓN}

Existen diferentes rutas de síntesis para la fabricación de nanofibras de polímero (ver Figura 2). A continuación, se explican cada una de estas rutas. 
Figura 1. Número de publicaciones con la palabra electrospinning

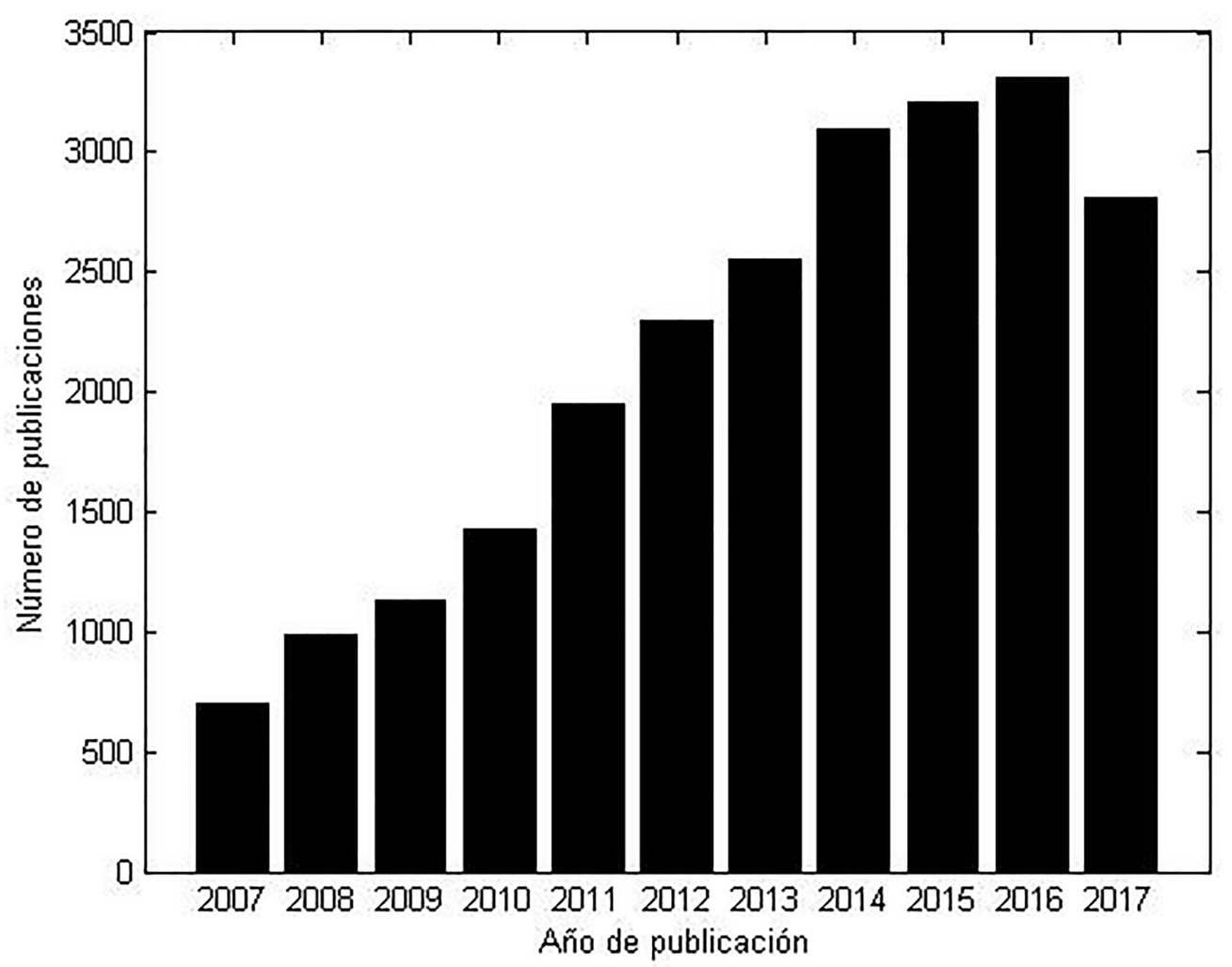

Fuente: Scopus (2017).

Figura 2. Rutas de síntesis para la fabricación de nanofibras.

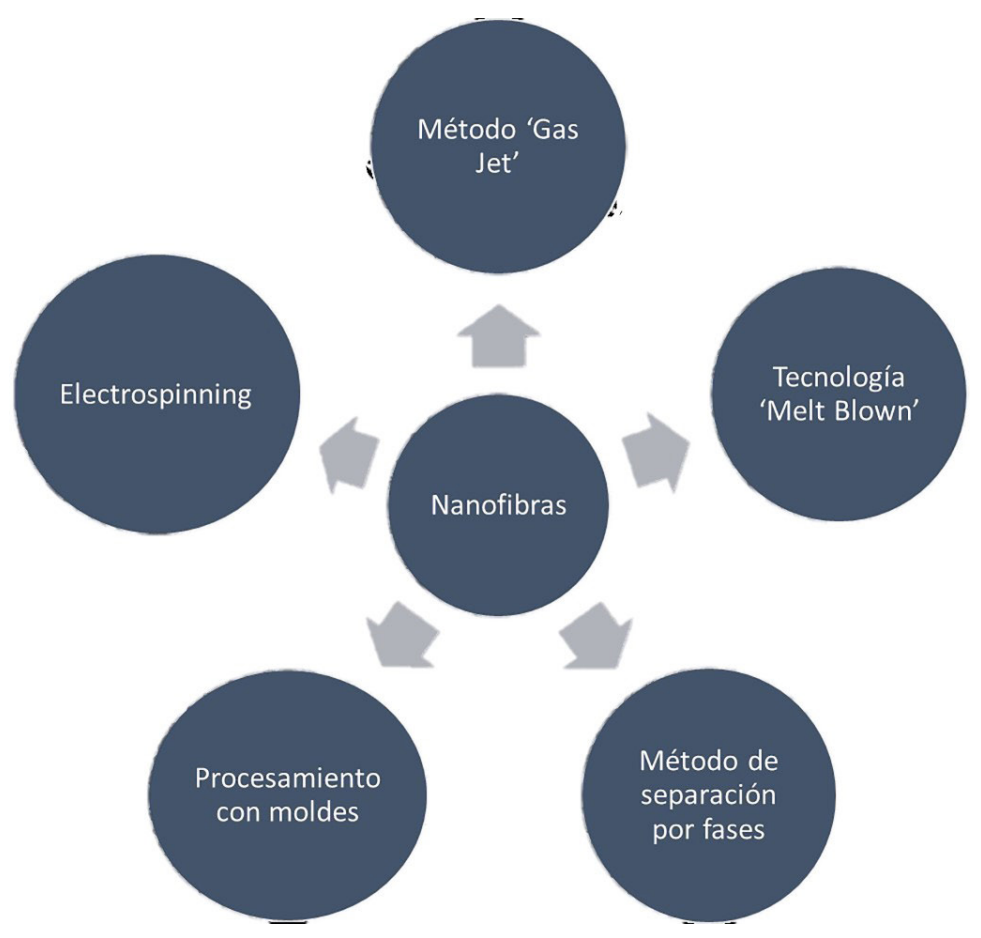

Fuente: elaboración propia. 


\section{Método gas jet}

El procesamiento de nanofibras por el método gas jet consiste en varios pasos: primero, la solución polimérica se lleva a una tobera y se extrude a flujo constante por medio de una bomba; cuando la solución polimérica llega al final del capilar acoplado a la tobera, se encuentra con un flujo constante de gas (generalmente aire) proveniente de un sistema de expansión de gas; a continuación, el gas evapora el solvente y se genera un hilo, que finalmente se recolecta a unos cuantos centímetros para su posterior uso (Benavides, Jana y Reneker, 2012; Reneker, 2003, 2004; Reneker, Chun y Ertley, 2002) (ver Figura $3)$. Con esta técnica se obtienen fibras de tamaño micrométrico y nanométrico, los cuales dependen de la capacidad de evaporación del solvente, la velocidad y la presión del gas, entre otros factores (Benavides, Jana y Reneker, 2013).

Figura 3. Esquema de producción de nanofibras con el método gas jet.

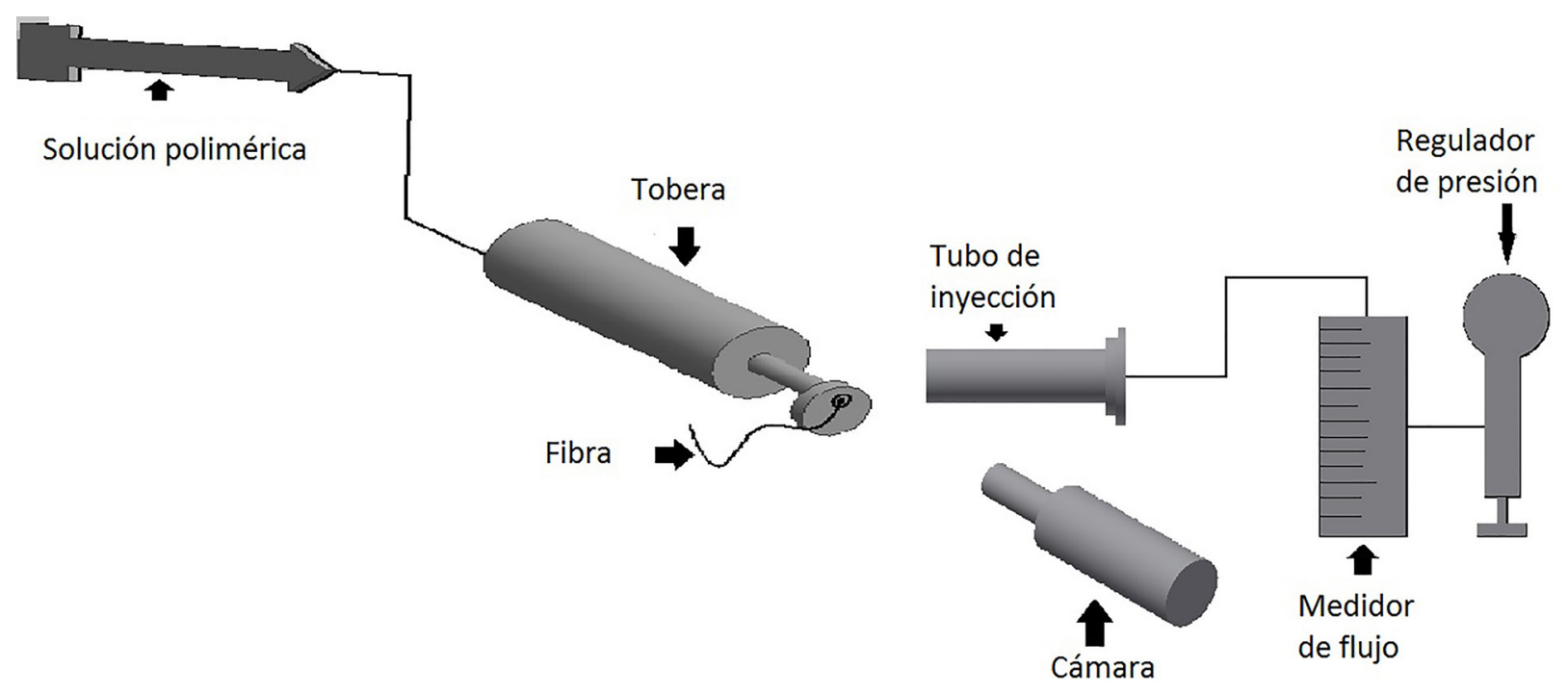

Fuente: elaboración propia.

\section{Tecnología melt blown}

El procesamiento de nanofibras mediante la tecnología melt blown consiste en la extrusión de un polímero fundido a través de un orificio: cuando la primera gota de polímero sale por dicho orificio, un flujo de gas caliente (generalmente aire) se suministra a $45^{\circ} \mathrm{C}$ por encima y por debajo (ver Figura 4), produciendo así un cono y consecutivamente el hilo, el cual es recolectado en un colector (Ellison, Phatak, Giles, Macosko y Bates, 2007). Esta técnica permite la fabricación de fibras de tamaño nanométrico $y$, además, es amigable con el medio ambiente (Nayak, 2017).

\section{Método de separación por fases}

La producción de fibras por el método de separación por fases consiste en proporcionar a la solución polimérica una serie de condiciones termodinámicas no favorables para la estabilidad, como exponerla a otro solvente inmiscible o disminuir la temperatura por debajo de la curva binodal de solubilidad; estas condiciones no favorables hacen que el polímero tome una ruta para establecer su equilibrio con el sistema. Esta ruta resulta en la formación de estructuras nanométricas como las nanofibras, que se obtienen una vez se elimina el solvente (He, Nie y Feng, 2014) (ver Figura 5). Por lo general, esta técnica se emplea en la fabricación de nanofibras naturales con miras a ser aplicadas en la ingeniería de tejidos (Ma y Zhang, 1999).

\section{Procesamiento con moldes}

El procesamiento con moldes es una técnica efectiva para controlar la morfología en la producción de materiales a escala nanométrica (Emre Kiyak 
Figura 4. Esquema de producción de nanofibras con la tecnología melt blown.

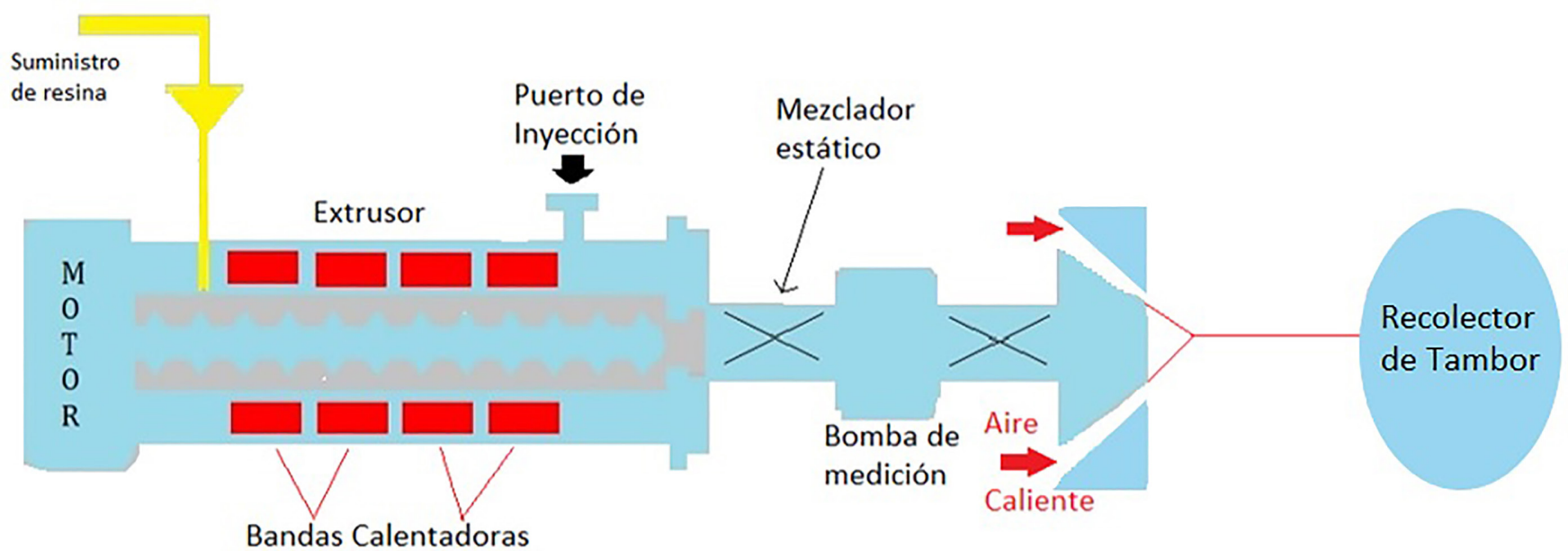

Fuente: elaboración propia.

Figura 5. Esquema de producción de nanofibras con el método de separación por fases.

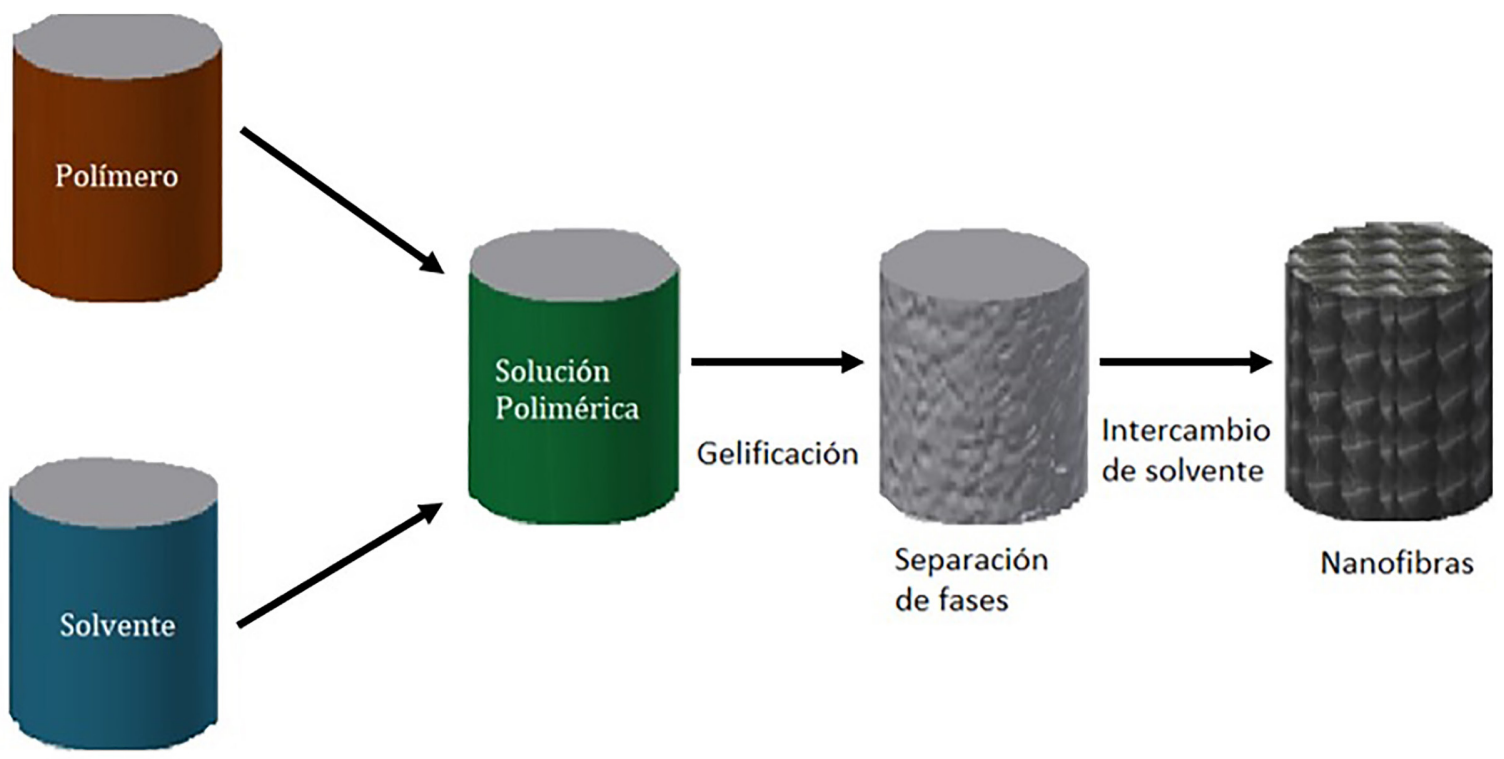

Fuente: elaboración propia.

y Cakmak, 2014; Wang y Qi, 2008). Esta técnica proporciona un tamaño adecuado del material que se quiere producir, por cuanto el molde facilita que se rellenen las estructuras con el elemento apropiado; de esta manera, se obtienen diferentes tipos de materiales unidimensionales como nanotubos, nanofibras, nanocintas, entre otros (Al-Kaysi et al., 2009; Liang et al., 2016) (ver Figura 6). Aunque esta ruta de síntesis es atractiva porque permite controlar el tamaño de las estructuras formadas (Naik et al., 2009), es costosa porque los moldes de los precursores usados se obtienen por métodos de morfosíntesis como la litografía (Jiao et al., 2006; Son et al., 2004; Xia et al., 2003); otra desventaja del proceso radica en la dificultad de deshacer el molde para obtener finalmente la estructura (Wang y Qi, 2008). 
Figura 6. Esquema de producción de nanofibras con la técnica de procesamiento con moldes.
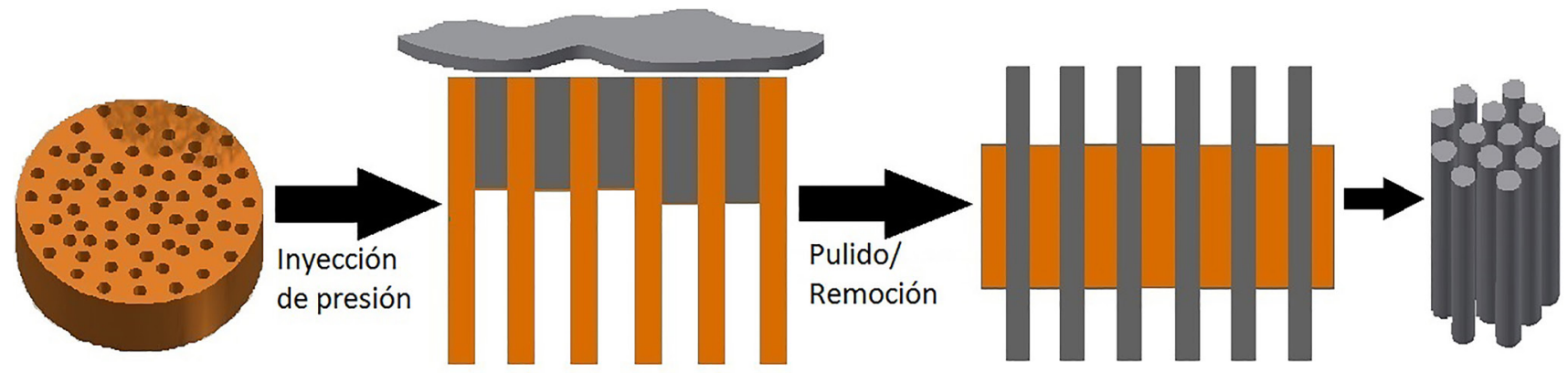

Fuente: elaboración propia.

\section{Técnica de electrospinning}

La técnica de electrospinning consiste en la creación de un cono, llamado cono de Taylor, debido a la acción de las fuerzas eléctricas sobre la superficie de un polímero del cual se obtienen las nanofibras y el colector utilizado (Deitzel, 2002; El-Newehy, AlDeyab, Kenawy, y Abdel-Megeed, 2011; Nirmala, Navamathavan, El-Newehy y Kim, 2011; Pedicini y Farris, 2004; Reneker y Chun, 1996; Yuan, Zhang, Dong y Sheng, 2004) (ver Figura 7). En este proceso se pueden emplear tanto polímeros naturales y sintéticos como mezclas de polímeros y polímeros funcionalizados con diferentes materiales, lo cual permite un amplio rango de diversas aplicaciones ( $\mathrm{Li}$ y Wang, 2013b).
Las características de las fibras obtenidas mediante electrospinning tienen gran potencial en aplicaciones como filtración, prendas protectoras, ingeniería de tejidos, apósitos para heridas, sistemas de liberación controlada de medicamentos, sensores y materiales ópticos, entre otros (Damodar, You y Chou, 2009; Rajesh, Ahuja y Kumar, 2009; Yoo, Kim y Park, 2009; Yang, Both, Yang, Walboomers y Jansen, 2009). El uso de polímeros funcionalizados con nanopartículas o diferentes materiales permiten que la matriz polimérica electrohilada se aplique en distintos ámbitos, por ejemplo: en catálisis, cuando se agregan nanopartículas o nanotubos de $\mathrm{TiO}_{2}$, así como en propiedades antimicrobianas y protectoras de rayos UV, cuando se agrega grafeno (Pant et al., 2012).

Figura 7. Esquema de producción de nanofibras con la técnica de electrospinning.

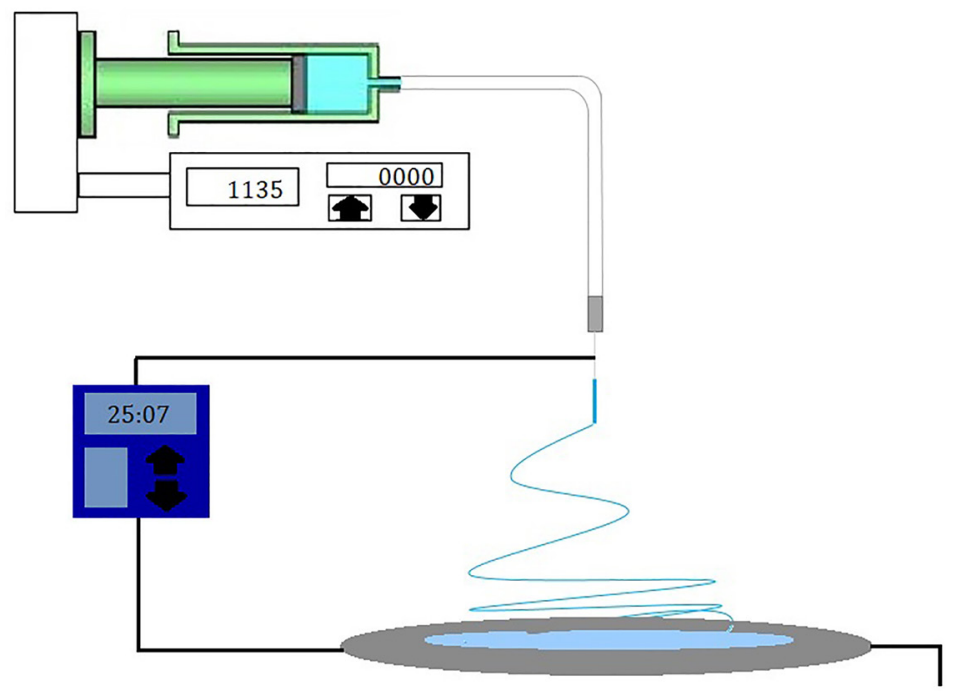


En la Tabla 2 se comparan las diferentes rutas de síntesis para la producción de nanofibras y se incluyen tanto las ventajas como las desventajas de cada método. A partir de esta información, se deduce que el electrospinning es una de las técnicas más atractivas.

Tabla 2. Comparación de las rutas de síntesis para la producción de nanofibras

\begin{tabular}{|c|c|c|c|c|}
\hline Proceso & $\begin{array}{l}\text { Facilidad de } \\
\text { procesamiento }\end{array}$ & Ventajas & Desventajas & Referencias \\
\hline Método gas jet & Fácil & $\begin{array}{l}\text { Uso de aire como gas impulsor. Posibilidad } \\
\text { de usar varios polímeros. }\end{array}$ & $\begin{array}{c}\text { Problemas en el ajuste de } \\
\text { la presión y el flujo de gas } \\
\text { óptimo. }\end{array}$ & $\begin{array}{l}\text { (Benavides et al., 2012; } \\
\text { Ghosh y Jana, 2015) }\end{array}$ \\
\hline $\begin{array}{l}\text { Tecnología } \\
\text { melt blown }\end{array}$ & Fácil & $\begin{array}{l}\text { Capacidad de manejo de muchos } \\
\text { polímeros, así como mezclas de estos. No } \\
\text { hay problemas por manejo de solventes. }\end{array}$ & $\begin{array}{l}\text { Proceso apto únicamente } \\
\text { para polímeros } \\
\text { termoplásticos. }\end{array}$ & (Bhat y Malkan, 2002) \\
\hline $\begin{array}{l}\text { Método de } \\
\text { separación } \\
\text { por fases }\end{array}$ & Fácil & $\begin{array}{l}\text { Posibilidad de adaptar propiedades } \\
\text { mecánicas. }\end{array}$ & $\begin{array}{l}\text { Bajo rendimiento. Limitado } \\
\text { a ciertos polímeros. }\end{array}$ & (Poole y Owens, 2003) \\
\hline $\begin{array}{l}\text { Procesamiento } \\
\text { con moldes }\end{array}$ & Difícil & $\begin{array}{l}\text { Controlabilidad sobre la morfología del } \\
\text { material obtenido. Materiales específicos } \\
\text { y viables para aplicaciones en el campo } \\
\text { biomédico. }\end{array}$ & $\begin{array}{l}\text { Producción costosa por } \\
\text { el uso de moldes que se } \\
\text { deben fabricar con base en } \\
\text { métodos como la litografía }\end{array}$ & (Vasita y Katti, 2006) \\
\hline $\begin{array}{l}\text { Técnica de } \\
\text { electrospinning }\end{array}$ & Fácil & $\begin{array}{c}\text { Económico. Fibras continuas. Facilidad } \\
\text { de funcionalización. Posibilidad de fibras } \\
\text { alineadas. }\end{array}$ & $\begin{array}{l}\text { Uso de solventes orgánicos } \\
\text { nocivos para la salud } \\
\text { y el medio ambiente. } \\
\text { Restricción en la } \\
\text { controlabilidad. }\end{array}$ & $\begin{array}{l}\text { (Kamiyama, Soeda, } \\
\text { Nagajima y Tanaka, 2012; } \\
\text { Poole y Owens, 2003) }\end{array}$ \\
\hline
\end{tabular}

Fuente: elaboración propia.

\section{EFECTOS DE LAS VARIABLES DE OPERACIÓN}

La producción de nanofibras mediante el método de electrospinning está determinada por los parámetros operacionales del proceso, las características de la solución utilizada y los factores ambientales bajo los cuales se produce. A continuación, se profundizará sobre el efecto de cada uno de estos parámetros en la producción de nanofibras.

\section{Parámetros de procesamiento}

Voltaje. El voltaje desempeña un rol importante en este proceso, únicamente con un voltaje superior al umbral -o crítico- se genera un cono de Taylor que es expulsado hacia el colector empleado (Li y Wang, 2013a). El uso de altos voltajes facilita la formación de nanofibras de menor diámetro (Yuan et al., 2004) y hace que el producto obtenido tenga forma de telaraña (Pant et al., 2010) (ver Figura 8).

Caudal. El caudal es otro de los parámetros importantes en esta ruta de síntesis. En la literatura se menciona que un flujo pequeño (entre 0.1 y 1 $\mathrm{mL} / \mathrm{h}$ ) es apropiado porque permite que la solución se polarice $y$, así, se pueda formar adecuadamente el cono de Taylor (Buchko, Chen, Shen y Martin, 1999). Asimismo, un flujo pequeño permite que el solvente utilizado se evapore y el polímero salga en forma de hilo (Li y Wang, 2013a). En el estudio de Buchko et al. (1999), un flujo igual a $0.66 \mathrm{~mL} / \mathrm{h}$ en la producción de nanofibras de polisulfona (PSF) proporcionó diámetros aún más pequeños que los valores mencionados.

Distancia aguja-colector. Se ha probado que la distancia entre la aguja y el colector influye en el diámetro de la fibra obtenida (Ki et al., 2005). Si la distancia es muy pequeña, la formación de nanofibras se ve afectada debido a que el solvente no tiene suficiente tiempo para evaporarse; por el contrario, si la distancia es muy grande, las fibras tienden a formar gotas que afectan su uniformidad (Li y Wang, 2013a). Por esta razón, es importante usar un método de optimización como lo hacen Yuan et al. (2004) en su estudio, donde concluyen que los parámetros más importantes en el procesamiento y fabricación de 
Figura 8. Formación de nanofibras de nylon con una configuración tipo telaraña, donde (A) es la micrografía obtenida con voltaje $=29 \mathrm{kV}$, flujo $=3 \mathrm{~mL} / \mathrm{h}$ y distancia ajuga-colector $=9 \mathrm{~cm}$, y (B) es la distribución del diámetro promedio de las nanofibras obtenidas.

(A)

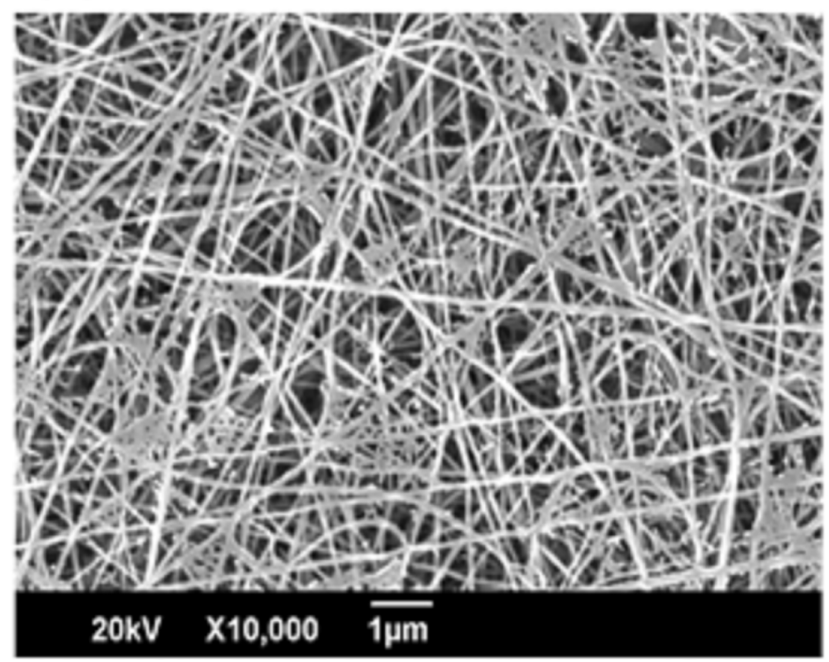

(B)

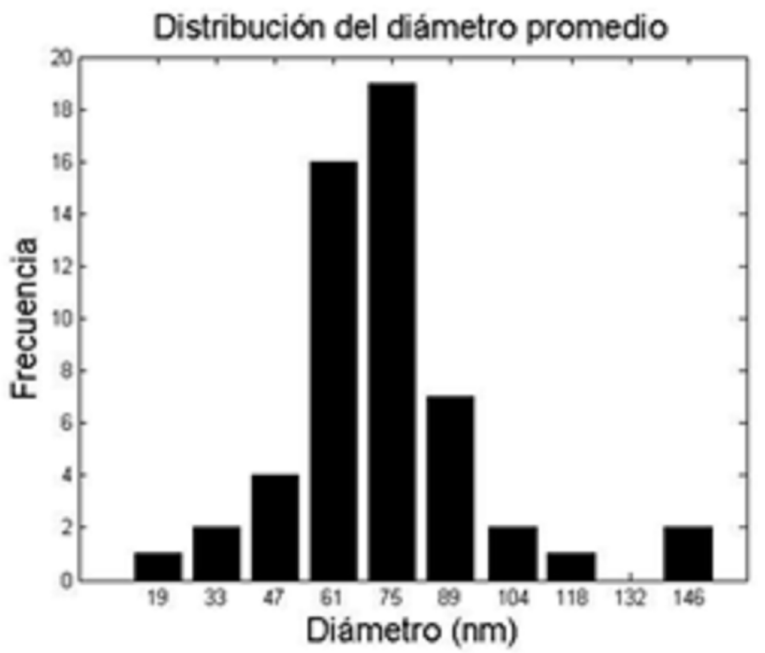

Fuente: elaboración propia.

nanofibras son: la distancia aguja-colector, el caudal, el voltaje y la concentración de la solución utilizada.

Colector. Los colectores usados durante el proceso de electrospinning son importantes porque son el sustrato donde se depositan las fibras. Las características de los colectores están determinadas por varios factores; sin embargo, se recomienda que sean superficies conductoras (generalmente aluminio) para que la descarga de alto voltaje se realice adecuadamente; además, el colector usado debe desprenderse rápidamente de la fibra una vez esta se ha depositado allí. Los colectores que se utilizan actualmente son: mallas (Wang et al., 2005), cuadrículas (Sundaray et al., 2004), tambores rotatorios o varillas giratorias (Xu, Inai, Kotaki y Ramakrishna, 2004), baños líquidos (Ki et al., 2007), entre otros.

\section{Parámetros de la solución}

Concentración y polímero. Este método permite la producción de nanofibras de diferentes polímeros, siempre y cuando estos puedan solubilizarse. En la Figura 9 se comparan nanofibras de nylon y PVP obtenidas mediante el proceso de electrospinning. La concentración de la solución empleada es de gran importancia: cuando la concentración es muy baja, se obtienen micro o nanopartículas y, si se aumenta un poco la concentración, aparecen nanofibras con gotas, es decir, fibras no uniformes; si la concentración es adecuada, las nanofibras se producen continuamente, pero, si es muy alta, no hay producción de nanofibras (Li y Wang, 2013a).

Peso molecular y viscosidad. Es importante conocer el peso molecular del polímero usado, ya que determina indirectamente la viscosidad de la solución debido a las cadenas de los enlaces poliméricos (Li y Wang, 2013a). Por tanto, la viscosidad debe tenerse en cuenta tanto en el bombeo o impulso del polímero como en la formación del cono de Taylor, dado que una viscosidad muy elevada no permite que se genere el cono, ocasiona la expulsión de polímero por la aguja e impide la creación de las fibras. Por otro lado, si la viscosidad es muy baja, no se pueden obtener fibras continuas y uniformes, por lo que es necesario ajustar el valor de la viscosidad (y de la concentración) para conseguir el tamaño y las características morfológicas adecuadas de las fibras (Sukigara, Gandhi, Ayutsede, Micklus y Ko, 2003). 
Figura 9. Micrografía SEM de nanofibras (A) de PVP con voltaje $=27 \mathrm{kV}$, flujo $=5 \mathrm{~mL} / \mathrm{h}$ y distancia aguja-colector $=9 \mathrm{~cm}, \mathrm{y}$ (B) de nylon con voltaje $=29 \mathrm{kV}$, flujo $=1 \mathrm{~mL} / \mathrm{h}$ y distancia aguja-colector $=13 \mathrm{~cm}$.

(A)

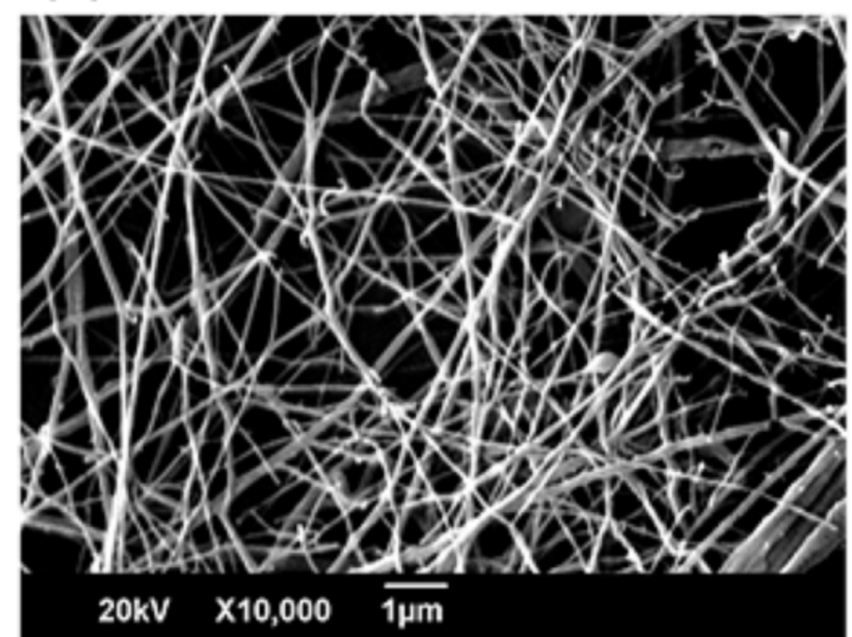

(B)

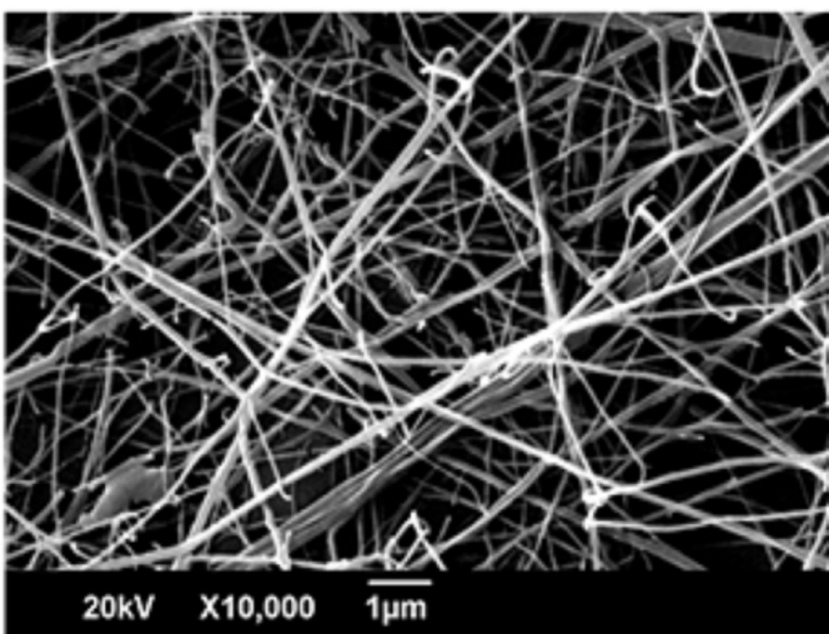

Fuente: elaboración propia

\section{Parámetros ambientales}

Temperatura y humedad. Los parámetros ambientales más importantes a tener en cuenta en la producción de nanofibras de polímero con electrospinning son la temperatura y la humedad. Se ha demostrado que, a medida que la temperatura incrementa, el diámetro de las fibras (fibras de poliamida-6) disminuye; el rango de temperatura adecuado para la fabricación de fibras de este polímero va desde $20^{\circ} \mathrm{C}$ (ambiente) hasta $60{ }^{\circ} \mathrm{C}$ (Mit-uppatham, Nithitanakul y Supaphol, 2004). En cuanto a la humedad, una poca cantidad de vapor de agua en el ambiente hace que el solvente pueda evaporarse más rápido, lo cual facilita la formación de las fibras; sin embargo, cuando la humedad del ambiente es alta, el diámetro de las nanofibras aumenta porque se neutralizan las cargas sobre la superficie del cono de Taylor, haciendo que las fuerzas eléctricas disminuyan; el rango de humedad adecuado para la fabricación de nanofibras está entre $0 \%$ y $50 \%$ (Casper, Stephens, Tassi, Chase y Rabolt, 2003).

\section{APLICACIONES}

La técnica de electrospinning tiene una gran variedad de aplicaciones en la industria debido a su versatilidad en cuanto a formas y materiales. A continuación, se presentan las aplicaciones que han sido más estudiadas, las cuales van desde la ingeniería de tejidos hasta los alimentos.

\section{Ingeniería de tejidos}

Diferentes polímeros naturales y sintéticos electrohilados se han empleado como scaffolds (o andamios celulares) en la regeneración de ciertos tejidos especializados, entre los que se encuentran:

- Piel: polímeros empleados como matrices extracelulares (ECM) transitorias para la regeneración cutánea en quemaduras o heridas, protegiendo de infecciones y de pérdida de fluidos (Braghirolli, Steffens y Pranke, 2014; Van der Veen, Boekema, Ulrich y Middelkoop, 2011).

- Nervio: polímeros empleados como uniones entre los puntos terminales de un nervio afectado por un corte o trauma físico, de manera que se pueda realizar reconexión unidireccional y crecimiento orientado de los axones y las células de Schwann (Braghirolli et al., 2014; Masaeli et al., 2013).

- Vasos sanguíneos: polímeros empleados para guiar el contacto y la adhesión de las células hospederas, incrementando la respuesta biológica 
y la regeneración vascular (Braghirolli et al., 2014; Mrówczyński et al., 2014).

- Cartílago: polímeros empleados como scaffolds para el sembrado celular de condrocitos y la regeneración de tejido cartilaginoso, el cual se caracteriza por presentar un comportamiento anisotrópico debido a la orientación y organización de la matriz extracelular.

- Hueso: polímeros empleados como facilitadores de reparación de tejido óseo afectado por traumas o enfermedades, y habitualmente funcionalizados con factores osteogénicos $\mathrm{y} / \mathrm{o}$ materiales nanoestucturados, como hidroxiapatita, que permiten tener resistencia mecánica y diferenciación celular osteogénica (Braghirolli et al., 2014; Jose et al., 2010; Lee, Rim, Jung y Shin, 2010).

- Columna vertebral: polímeros empleados para guiar la regeneración espontánea de neuronas y axones; sin embargo, los métodos efectivos para su reconstrucción son muy escasos y no han permitido contrarrestar totalmente el déficit permanente funcional y neurológico causado por trauma, degradación o inflamación del tejido, o necrosis celular (Braghirolli et al., 2014; Du et al., 2014; Liu et al., 2013).

Adicionalmente, la técnica de electrospinning ha sido empleada en la fabricación de scaffolds que, además de mostrar resistencia mecánica y estar diseñados con precisión, permiten actuar de manera local, selectiva y controlada. Si bien se deben considerar muchas variables, como la solubilidad y estabilidad del agente a ser encapsulado, también se debe tener en cuenta la naturaleza y estructura de la fibra, de manera que permita una liberación que evite concentraciones tóxicas que vayan al torrente sanguíneo (Khalf y Madihally, 2017; Kruger, Benck, Singer y Kramer, 2012)

\section{Energía}

Entre las tecnologías que producen energía libre de carbono, se encuentran las celdas de combustible de óxido sólido (SOFC, por sus siglas en inglés), las cuales han sido la tecnología líder en las últimas décadas. Las SOFC son sistemas de conversión de energía que convierten los combustibles directamente en energía a través de reacciones electroquímicas. Como las celdas de combustible evitan el proceso de combustión que prevalece en las tecnologías convencionales de producción de energía, emiten mucho menos carbono (Pintauro et al., 2007; Tsipis y Kharton, 2008).

Las SOFC tienen una eficiencia muy alta que generalmente aumenta cuando se introduce la cogeneración; su principal ventaja es la flexibilidad en cuanto a combustible, ya que puede usar hidrógeno o cualquier hidrocarburo. Una SOFC consiste en un ánodo, un cátodo y un electrolito; un combustible y un oxidante se introducen en el ánodo y el cátodo, respectivamente; la reacción electroquímica tiene lugar debido a la diferencia en el potencial químico a través del electrolito sólido, el cual se selecciona de modo que solo permite que los iones de óxido lo atraviesen y bloqueen los electrones; por lo tanto, se utiliza un circuito externo para aprovechar esta concentración de electrones (Pintauro et al., 2007; Tsipis y Kharton, 2008).

Desde hace varios años, el electrohilado se ha utilizado en las SOFC para dar soporte con nanofibras de polímero, brindando continuidad junto con una serie de métodos innovadores como el prensado isostático en frío, la extrusión y la fundición en gel (Aruna, Balaji, Kumar y Prakash, 2017; Orera, Laguna-Bercero y Larrea, 2014). La producción de energía limpia ha sido el principal campo de investigación durante este siglo y probablemente lo seguirá siendo. Se ha evidenciado una creciente preocupación por el medio ambiente, así como una intensa contaminación, en la cual la energía es determinante debido a la generación de gases de efecto invernadero. Por esto, la necesidad de producir energía limpia se ha vuelto más importante que nunca (Aruna et al., 2017).

La técnica de electrohilado es un método eficiente y prometedor en la producción de nanofibras. Si se utiliza adecuadamente, tendrá un gran impacto en cómo se fabrican los electrodos SOFC, en los que actualmente predomina la cerámica en polvo. Dado que se trata de una técnica de producción rentable que proporciona una microestructura muy favorable en términos de porosidad continua y sitios de reacción relativamente más altos que con electrodos en polvo, las nanofibras han mejorado significativamente el rendimiento de las SOFC, duplicando la densidad de potencia y reduciendo su polarización en medio de técnicas de química verde (Aruna et al., 2017). 


\section{Medio ambiente}

La creciente preocupación por el cambio climático y la contaminación hídrica y atmosférica ha suscitado estudios exhaustivos sobre la posibilidad de descontaminar las fuentes acuíferas por medio de métodos fotocatalíticos, con diferentes catalizadores sostenidos sobre matrices poliméricas electrohiladas (Patil et al., 2017). Yang et al. (2013) desarrollaron un compuesto de polipirrol con $\mathrm{Ag}-\mathrm{TiO}_{2}$ y demostraron una capacidad fotocatalítica interesante, que puede utilizarse en el tratamiento de aguas. Otros estudios que usan nanopartículas, nanotubos y nanohojas de $\mathrm{TiO}_{2}$ o de $\mathrm{ZnO}$ en diferentes estados cristalinos, como rutilo o anatasa, aportan a una solución efectiva de la degradación de compuestos orgánicos en el agua (Ganesh, Nair, Raut, Walsh y Ramakrishna, 2012; Lai, Wang, Zhao, Fong y Zhu, 2013; Lang, Wu y Xu, 2012; Meng et al., 2011).

Además, las nanofibras obtenidas mediante el proceso de electrospinning tienen la capacidad de estar en sistemas de filtración de alta eficiencia, los cuales retienen compuestos o elementos como metales pesados (Celebioglu, Yildiz y Uyar, 2017; Faccini et al., 2015). Por ejemplo, los valores de eficiencia obtenidos en fibras electrohiladas de nylon- 6 son del orden de 99.993\%; mientras que, en filtros comerciales, esta eficiencia es de aproximadamente $99.97 \%$ (Barhate y Ramakrishna, 2007). Estos sistemas pueden emplearse para gases o líquidos; en el caso de aguas residuales obtenidas en plantas de tratamiento, las nanofibras son funcionalizadas con nanopartículas para reducir la actividad microbiana patógena (Lev, Holba, Kalhotka, Mikula y Kimmer, 2012).

Por otro lado, los sistemas de sensores se basan en un circuito electrónico que puede detectar cambios físicos y químicos en su superficie debido a la absorción de un estimulante químico; estos estimulantes modifican las propiedades eléctricas del circuito (sensor) y, de esta forma, se pueden correlacionar los cambios en las mediciones eléctricas con cantidades medibles (Patil et al., 2017). Recientemente, se han evaluado sensores basados en nanofibras de diferentes polímeros, los cuales se emplean en la detección de contaminantes de agua y suelo, como sensores de temperatura y de campo magnético, y en el control de emisiones y alarmas de gas (Kumar, Al-Dossary, Kumar y Umar, 2015).

\section{Alimentos}

El desperdicio y la protección de alimentos son problemas tanto económicos como sociales. En el sector de alimentos, las nanofibras tienen una aplicación de alto impacto en el empaque y conservación de estos. Dumitriu, Mitchell, Davis y Vasile (2017) desarrollaron estudios sobre la capacidad antioxidante de nanofibras de poli( $\alpha$ caprolactona) con vitamina $\mathrm{E}$ para usar en empaques alimenticios y demostraron que la gran área superficial de las nanofibras, junto con la adición del agente antioxidante (vitamina E), proporciona al material las características adecuadas para retardar el deterioro de los alimentos por acción enzimática y oxidante.

Otra aplicación interesante de las nanofibras en alimentos es la nanoencapsulación de componentes bioactivos (agentes bioactivos se encuentran dentro de las nanofibras), los cuales son moléculas que proveen beneficios a la salud humana como la prevención de aparición de enfermedades causadas por actividad oxidante, inflamatoria o cancerígena (Wen, Zong, Linhardt, Feng y Wu, 2017). Recientes estudios demuestran que la encapsulación de componentes, como enzimas, proteínas, probióticos, entre otros, es un medio eficiente porque protege a estas moléculas del oxígeno no deseado debido a reacciones de oxidación; además, la encapsulación permite mejorar la estabilidad de las moléculas bioactivas y la capacidad de incrementar la viabilidad de probióticos, los cuales son importantes para fabricar alimentos funcionales. Otra ventaja es la habilidad de enmascarar olores y sabores para mejorar la aceptación de un producto, así como el control sobre la preservación de los alimentos en la cadena de frío (Fabra, López-Rubio y Lagaron, 2016; Pérez-Masiá, López-Rubio, Fabra y Lagaron, 2014; Wen et al., 2017).

\section{CONCLUSIONES}

El proceso de electrospinning se caracteriza por ser simple, rentable y versátil para la producción de nanofibras; además, es atractivo porque proporciona fibras funcionales en el rango de micrómetros y nanómetros, lo cual permite tener características como alta relación superficie/volumen, adecuada porosidad y morfologías adaptables. Los parámetros de procesamiento, de solución y ambientales que influyen en la producción de nanofibras son de gran 
importancia, ya que estos deben responder a los requerimientos de las diferentes aplicaciones en cuanto a la morfología de las nanofibras. El uso de nanofibras en los campos de energía, ingeniería de tejidos, medio ambiente $\mathrm{y}$ alimentos tiene un gran potencial para la solución de problemáticas de alto impacto. Por último, persisten retos acerca del uso de fibras electrohiladas, relacionados con el modelamiento bajo diferentes parámetros y las aplicaciones tanto en alimentos como en medicina para la liberación controlada de nutrientes y medicamentos, respectivamente.

\section{REFERENCIAS}

Ahn, Y. C., Park, S. K., Kim, G. T., Hwang, Y. J., Lee, C. G., Shin, H. S. y Lee, J. K. (2006). Development of high efficiency nanofilters made of nanofibers. Current Applied Physics, 6(6), 1030-1035. doi: 10.1016/j. cap.2005.07.013

Al-Kaysi, R. O., Ghaddar, T. H. y Guirado, G. (2009). Fabrication of One-Dimensional Organic Nanostructures Using Anodic Aluminum Oxide Templates. Journal of Nanomaterials, 2009, 1-14. doi: 10.1155/2009/436375

Alivisatos, P., Barbara, P. F., Castleman, A. W., Chang, J., Dixon, D. A., Klein, M. L., ... Thompson, M. E. (1998). From Molecules to Materials: Current Trends and Future Directions. Advanced Materials, 10(16), 1297-1336. doi: 10.1002/ (SICI)1521-4095(199811)10:16<1297::AIDADMA1297>3.0.CO;2-7

Aruna, S. T., Balaji, L. S., Kumar, S. S. y Prakash, B. S. (2017). Electrospinning in solid oxide fuel cells - A review. Renewable and Sustainable Energy Reviews, 67, 673-682. doi: 10.1016/j.rser.2016.09.003

Barhate, R. S. y Ramakrishna, S. (2007). Nanofibrous filtering media: Filtration problems and solutions from tiny materials. Journal of Membrane Science, 296(1), 1-8. doi: 10.1016/j.memsci.2007.03.038

Benavides, R. E., Jana, S. C. y Reneker, D. H. (2012). Nanofibers from Scalable Gas Jet Process. ACS Macro Letters, 1(8), 1032-1036. doi: 10.1021/ mz300297g
Benavides, R. E., Jana, S. C. y Reneker, D. H. (2013). Role of Liquid Jet Stretching and Bending Instability in Nanofiber Formation by Gas Jet Method. Macromolecules, 46(15), 6081-6090. doi: 10.1021/ ma400900s

Bhat, G. S. y Malkan, S. R. (2002). Extruded continuous filament nonwovens: Advances in scientific aspects. Journal of Applied Polymer Science, 83(3), 572-585. doi: 10.1002/app.2259

Braghirolli, D. I., Steffens, D. y Pranke, P. (2014). Electrospinning for regenerative medicine: a review of the main topics. Drug Discovery Today, 19(6), 743-753. doi: 10.1016/j.drudis.2014.03.024

Buchko, C. J., Chen, L. C., Shen, Y. y Martin, D. C. (1999). Processing and microstructural characterization of porous biocompatible protein polymer thin films. Polymer, 40(26), 7397-7407. doi: 10.1016/S00323861(98)00866-0

Casper, C. L., Stephens, J. S., Tassi, N. G., Chase, D. B. y Rabolt. J. F. (2003). Controlling Surface Morphology of Electrospun Polystyrene Fibers: Effect of Humidity and Molecular Weight in the Electrospinning Process. Macromolecules, 37(2), 573-578. doi: 10.1021/MA0351975

Celebioglu, A., Yildiz, Z. I. y Uyar, T. (2017). Electrospun crosslinked poly-cyclodextrin nanofibers: Highly efficient molecular filtration thru host-guest inclusion complexation. Scientific Reports, 7. doi: 10.1038/s41598-017-07547-4

Da Silva Vaz, B., Vieira Costa, J. A. y De Morais, M. G. (2017). Production of polymeric nanofibers with different conditions of the electrospinning process. Matéria (Rio de Janeiro), 22(2). doi: 10.1590/ s1517-707620170002.0180

Damodar, R. A., You, S.-J. y Chou, H.-H. (2009). Study the self cleaning, antibacterial and photocatalytic properties of $\mathrm{TiO}_{2}$ entrapped PVDF membranes. Journal of Hazardous Materials, 172(2-3), 13211328. doi: 10.1016/j.jhazmat.2009.07.139

Deitzel, J. (2002). Electrospinning of polymer nanofibers with specific surface chemistry. Polymer, 43(3), 1025-1029. doi: 10.1016/S0032-3861(01)00594-8 
Doshi, J. y Reneker, D. H. (1995). Electrospinning process and applications of electrospun fibers. Journal of Electrostatics, 35(2-3), 151-160. doi: 10.1016/0304-3886(95)00041-8

Du, B., Zeng, C., Zhang, W., Quan, D., Ling, E. y Zeng, Y. (2014). A comparative study of gelatin sponge scaffolds and PLGA scaffolds transplanted to completely transected spinal cord of rat. Journal of Biomedical Materials Research Part A, 102(6), 1715-1725. doi: 10.1002/jbm.a.34835

Dumitriu, R. P., Mitchell, G. R., Davis, F. J. y Vasile, C. (2017). Functionalized Coatings by Electrospinning for Anti-oxidant Food Packaging. Procedia Manufacturing, 12, 59-65. doi: 10.1016/j. promfg.2017.08.008

El-Newehy, M. H., Al-Deyab, S. S., Kenawy, E.-R. y AbdelMegeed, A. (2011). Nanospider Technology for the Production of Nylon-6 Nanofibers for Biomedical Applications. Journal of Nanomaterials, 2011, 1-8. doi: 10.1155/2011/626589

Ellison, C. J., Phatak, A., Giles, D. W., Macosko, C. W. y Bates, F. S. (2007). Melt blown nanofibers: Fiber diameter distributions and onset of fiber breakup. Polymer, 48(11), 3306-3316. doi: 10.1016/j. polymer.2007.04.005

Emre Kiyak, Y. y Cakmak, E. (2014). Nanofiber Production Methods. Electronic Journal of Textile Technologies /Tekstil Teknolojileri Elektronik Dergisi, 8(3), 49-60. Recuperado de http://teknolojikarastirmalar.com/ pdf/tr/04_2014_8_3_1169_1945.pdf

Fabra, M. J., López-Rubio, A. y Lagaron, J. M. (2016). Use of the electrohydrodynamic process to develop active/bioactive bilayer films for food packaging applications. Food Hydrocolloids, 55, 11-18. doi: 10.1016/j.foodhyd.2015.10.026

Faccini, M., Borja, G., Boerrigter, M., Morillo Martín, D., Martínez Crespiera, S., Vázquez-Campos, S., ... Amantia, D. (2015). Electrospun Carbon Nanofiber Membranes for Filtration of Nanoparticles from Water. Journal of Nanomaterials, 2015, 1-9. doi: $10.1155 / 2015 / 247471$

Ganesh, V. A., Nair, A. S., Raut, H. K., Walsh, T. M. y Ramakrishna, S. (2012). Photocatalytic superhydrophilic $\mathrm{TiO}_{2}$ coating on glass by electrospinning. RSC Advances, 2(5), 2067-2072. doi: 10.1039/c2ra00921h

Ghosh, M. y Jana, S. C. (2015). Bi-component inorganic oxide nanofibers from gas jet fiber spinning process. RSC Advances, 5(127), 105313-105318. doi: 10.1039/C5RA20963C

Haider, A., Haider, S. y Kang, I.-K. (2015). A comprehensive review summarizing the effect of electrospinning parameters and potential applications of nanofibers in biomedical and biotechnology. Arabian Journal of Chemistry. doi: 10.1016/j.arabjc.2015.11.015

He, C., Nie, W. y Feng, W. (2014). Engineering of biomimetic nanofibrous matrices for drug delivery and tissue engineering. Journal of Materials Chemistry B, 2(45), 7828-7848. doi: 10.1039/ C4TB01464B

Jiao, S., Xu, L., Jiang, K. y Xu, D. (2006). Well-Defined Non-spherical Copper Sulfide Mesocages with Single-Crystalline Shells by Shape-Controlled $\mathrm{Cu}_{2} \mathrm{O}$ Crystal Templating. Advanced Materials, 18(9), 1174-1177. doi: 10.1002/adma.200502386

Jose, M. V., Thomas, V., Xu, Y., Bellis, S., Nyairo, E. y Dean, D. (2010). Aligned Bioactive MultiComponent Nanofibrous Nanocomposite Scaffolds for Bone Tissue Engineering. Macromolecular Bioscience, 10(4), 433-444. doi: 10.1002/mabi.200900287

Kamiyama, M., Soeda, T., Nagajima, S. y Tanaka, K. (2012). Development and application of highstrength polyester nanofibers. Polymer Journal, 44(10), 987-994. doi: 10.1038/pj.2012.63

Khalf, A. y Madihally, S. V. (2017). Recent advances in multiaxial electrospinning for drug delivery. European Journal of Pharmaceutics and Biopharmaceutics, 112, 1-17. doi: 10.1016/j. ejpb.2016.11.010

Ki, C. S., Baek, D. H., Gang, K. D., Lee, K. H., Um, I. C. y Park, Y. H. (2005). Characterization of gelatin nanofiber prepared from gelatin-formic acid solution. Polymer, 46(14), 5094-5102. doi: 10.1016/j.polymer.2005.04.040 
Ki, C. S., Kim, J. W., Hyun, J. H., Lee, K. H., Hattori, M., Rah, D. K. y Park, Y. H. (2007). Electrospun threedimensional silk fibroin nanofibrous scaffold. Journal of Applied Polymer Science, 106(6), 39223928. doi: 10.1002/app.26914

Kruger, B., Benck, U., Singer, T.y Kramer, B. K. (2012). Druginduced impairment of renal function. Deutsche Medizinische Wochenschrift, 137(38), 1873-1877. doi: $10.1055 / \mathrm{s}-0032-1305312$

Kumar, R., Al-Dossary, O., Kumar, G. y Umar, A. (2015). Zinc Oxide Nanostructures for $\mathrm{NO}_{2}$ Gas-Sensor Applications: A Review. Nano-Micro Letters, 7(2), 97-120. doi: 10.1007/s40820-014-0023-3

Lai, C., Wang, X., Zhao, Y., Fong, H. y Zhu, Z. (2013). Effects of humidity on the ultraviolet nanosensors of aligned electrospun $\mathrm{ZnO}$ nanofibers. RSC Advances, 3(18), 6640-6645. doi: 10.1039/ c3ra23420g

Lang, L., Wu, D. y Xu, Z. (2012). Controllable Fabrication of $\mathrm{TiO}_{2}$ 1D-Nano/Micro Structures: Solid, Hollow, and Tube-in-Tube Fibers by Electrospinning and the Photocatalytic Performance. Chemistry - A European Journal, 18(34), 10661-10668. doi: 10.1002/chem.201200378

Lee, J. H., Rim, N. G., Jung, H. S. y Shin, H. (2010). Control of Osteogenic Differentiation and Mineralization of Human Mesenchymal Stem Cells on Composite Nanofibers Containing Poly[lactic- co -(glycolic acid)] and Hydroxyapatite. Macromolecular Bioscience, 10(2), 173-182. doi: 10.1002/mabi.200900169

Lev, J., Holba, M., Kalhotka, L., Mikula, P. y Kimmer, D. (2012). Improvements in the Structure of Electrospun Polyurethane Nanofibrous Materials Used for Bacterial Removal from Wastewater. International Journal of Theoretical and Applied Nanotechnology, 1(1), 16-20. doi: 10.11159/ ijtan.2012.003

Li, Z. y Wang, C. (2013a). Effects of Working Parameters on Electrospinning. En One-Dimensional nanostructures (pp. 15-28). Springer, Berlin, Heidelberg. doi: 10.1007/978-3-642-36427-3_2
Li, Z. y Wang, C. (2013b). Introduction of Electrospinning. En One-Dimensional nanostructures (pp. 1-13). Springer, Berlin, Heidelberg. doi: 10.1007/978-3642-36427-3_1

Liang, D., Hsiao, B. S. y Chu, B. (2007). Functional electrospun nanofibrous scaffolds for biomedical applications. Advanced Drug Delivery Reviews, 59(14), 1392-1412. doi: 10.1016/j.addr.2007.04.021

Liang, L., Kang, X., Sang, Y. y Liu, H. (2016). OneDimensional Ferroelectric Nanostructures: Synthesis, Properties, and Applications. Advanced Science, 3(7). doi: 10.1002/advs.201500358

Liu, J., Chen, Q., Zhang, Z., Zheng, Y., Sun, X., Cao, X., ... Jiang, P. (2013). Fibrin scaffolds containing ectomesenchymal stem cells enhance behavioral and histological improvement in a rat model of spinal cord injury. Cells Tissues Organs, 198(1), 3546. doi: 10.1159/000351665

Ma, P. X. y Zhang, R. (1999). Synthetic nano-scale fibrous extracellular matrix. Journal of Biomedical Materials Research, 46(1), 60-72. Recuperado de http://www.ncbi.nlm.nih.gov/pubmed/10357136

Masaeli, E., Morshed, M., Nasr-Esfahani, M. H., Sadri, S., Hilderink, J., Van Apeldoorn, A., ... Moroni, L. (2013). Fabrication, Characterization and Cellular Compatibility of Poly(Hydroxy Alkanoate) Composite Nanofibrous Scaffolds for Nerve Tissue Engineering. PLOS ONE, 8(2). doi: 10.1371/journal. pone.0057157

Meng, X., Shin, D.-W., Yu, S. M., Jung, J. H., Kim, H. I., Lee, H. M., ... Yoo, J.-B. (2011). Growth of hierarchical $\mathrm{TiO}_{2}$ nanostructures on anatase nanofibers and their application in photocatalytic activity. CrystEngComm, 13(8), 3021-3029. doi: 10.1039/c0ce00765j

Mit-uppatham, C., Nithitanakul, M. y Supaphol, P. (2004). Ultrafine Electrospun Polyamide-6 Fibers: Effect of Solution Conditions on Morphology and Average Fiber Diameter. Macromolecular Chemistry and Physics, 205(17), 2327-2338. doi: 10.1002/macp.200400225 
Mrówczyński, W., Mugnai, D., De Valence, S., Tille, J. C., Khabiri, E., Cikirikcioglu, M., ... Walpoth, B. H. (2014). Porcine carotid artery replacement with biodegradable electrospun poly-e-caprolactone vascular prosthesis. Journal of Vascular Surgery, 59(1), 210-219. doi: 10.1016/J.JVS.2013.03.004

Naik, N., Caves, J., Kumar, V., Chaikof, E. y Allen, M. G. (2009). A template-based fabrication technique for spatially-designed polymer micro/ nanofiber composites. Digest of Technical Papers. International Conference on Solid-State Sensors, Actuators, and Microsystems, 2009, 1869-1872. doi: 10.1109/SENSOR.2009.5285711

Nayak, R. (2017). Polypropylene nanofibers. Melt electrospinning versus meltblowing. Springer International Publishing. doi: 10.1007/978-3-31961458-8

Nirmala, R., Navamathavan, R., El-Newehy, M. H. y Kim, H. Y. (2011). Preparation and electrical characterization of polyamide-6/chitosan composite nanofibers via electrospinning. Materials Letters, 65(3), 493-496. doi: 10.1016/j. matlet.2010.10.066

Orera, V. M., Laguna-Bercero, M. A. y Larrea, A. (2014). Fabrication Methods and Performance in Fuel Cell and Steam Electrolysis Operation Modes of Small Tubular Solid Oxide Fuel Cells: A Review. Frontiers in Energy Research, 2(22). doi: 10.3389/ fenrg.2014.00022

Ozin, G. A. (1992). Nanochemistry: Synthesis in diminishing dimensions. Advanced Materials, 4(10), 612-649. doi: 10.1002/adma.19920041003

Pant, H. R., Bajgai, M. P., Yi, C., Nirmala, R., Nam, K. T., Baek, W. y Kim, H. Y. (2010). Effect of successive electrospinning and the strength of hydrogen bond on the morphology of electrospun nylon- 6 nanofibers. ColloidsandSurfacesA:Physicochemical and Engineering Aspects, 370(1-3), 87-94. doi: 10.1016/j.colsurfa.2010.08.051

Pant, H. R., Park, C. H., Tijing, L. D., Amarjargal, A., Lee, D.-H. y Kim, C. S. (2012). Bimodal fiber diameter distributed graphene oxide/nylon-6 composite nanofibrous mats via electrospinning. Colloids and Surfaces A: Physicochemical and
Engineering Aspects, 407, 121-125. doi: 10.1016/j. colsurfa.2012.05.018

Patil, J. V, Mali, S. S., Kamble, A. S., Hong, C. K., Kim, J. H. y Patil, P. S. (2017). Electrospinning: A versatile technique for making of $1 \mathrm{D}$ growth of nanostructured nanofibers and its applications: An experimental approach. Applied Surface Science, 423, 641-674. doi: 10.1016/j.apsusc.2017.06.116

Pedicini, A. y Farris, R. J. (2004). Thermally induced color change in electrospun fiber mats. Journal of Polymer Science Part B: Polymer Physics, 42(5), 752-757. doi: 10.1002/polb.10711

Pérez-Masiá, R., López-Rubio, A., Fabra, M. J. y Lagaron, J. M. (2014). Use of electrohydrodynamic processing to develop nanostructured materials for the preservation of the cold chain. Innovative Food Science \& Emerging Technologies, 26, 415423. doi: 10.1016/j.ifset.2014.10.010

Pintauro, P., Mather, P., Arnoult, O., Choi, J., Wycisk, R. y Lee, K. M. (2007). Composite Membranes for Hydrogen/Air PEM Fuel Cells. ECS Transactions, 11(1), 79-87. doi: 10.1149/1.2780917

Poole, C. P. y Owens, F. J. (2003). Introduction to nanotechnology. John Wiley \& Sons.

Rajesh, Ahuja, T. y Kumar, D. (2009). Recent progress in the development of nano-structured conducting polymers/nanocomposites for sensor applications. Sensors and Actuators B: Chemical, 136(1), 275286. doi: 10.1016/J.SNB.2008.09.014

Reneker, D. H. (2003). Process and apparatus for the production of nanofibers. Google Patents. Recuperado de https://www.google.ch/patents/ US6520425

Reneker, D. H. (2004). Process and apparatus for the production of nanofibers. Google Patents. Recuperado de http://www.google.ch/patents/ US6695992

Reneker, D. H. y Chun, I. (1996). Nanometre diameter fibres of polymer, produced by electrospinning. Nanotechnology, 7(3). 
Reneker, D. H., Chun, I. y Ertley, D. (2002). Process and apparatus for the production of nanofibers. Google Patents. Recuperado de https://www.google.ch/ patents/US6382526

Schulz, W. (2000). Nanotechnology: The next big thing. Chemical and Engineering News, 78(18), 41-47. doi: 10.1021/cen-v078n018.p041

Scopus. (2017). Journal title list. Recuperado de https:// www.scopus.com

Son, D. H., Hughes, S. M., Yin, Y. y Alivisatos, A. Paul (2004). Cation Exchange Reactions in Ionic Nanocrystals. Science, 306(5698), 1009-1012. doi: $10.1126 /$ science. 1103755

Sukigara, S., Gandhi, M., Ayutsede, J., Micklus, M. y Ko, F. (2003). Regeneration of Bombyx mori silk by electrospinning-part 1: processing parameters and geometric properties. Polymer, 44(19), 57215727. doi: 10.1016/S0032-3861(03)00532-9

Sundaray, B., Subramanian, V., Natarajan, T. S., Xiang, R.-Z., Chang, C.-C. y Fann, W.-S. (2004). Electrospinning of continuous aligned polymer fibers. Applied Physics Letters, 84(7), 1222-1224. doi: $10.1063 / 1.1647685$

Taylor, G. (1969). Electrically Driven Jets. Proceedings of the Royal Society A: Mathematical, Physical and Engineering Sciences, 313(1515), 453-475. doi: 10.1098/rspa.1969.0205

Tsipis, E. V. y Kharton, V. V. (2008). Electrode materials and reaction mechanisms in solid oxide fuel cells: a brief review. Journal of Solid State Electrochemistry, 12(11), 1367-1391. doi: 10.1007/s10008-0080611-6

Van der Veen, V. C., Boekema, B. K. H. L., Ulrich, M. M. W. y Middelkoop, E. (2011). New dermal substitutes. Wound Repair and Regeneration, 19(s1), s59-s65. doi: 10.1111/j.1524-475X.2011.00713.x

Vasita, R. y Katti, D. S. (2006). Nanofibers and their applications in tissue engineering. International Journal of Nanomedicine, 1(1), 15-30. Recuperado de http://www.ncbi.nlm.nih.gov/ pubmed/17722259
Wang, G., Shen, X., Yao, J. y Park, J. (2009). Graphene nanosheets for enhanced lithium storage in lithium ion batteries. Carbon, 47(8), 2049-2053. doi: 10.1016/j.carbon.2009.03.053

Wang, H. y Qi, L. (2008). Controlled Synthesis of $\mathrm{Ag}_{2} \mathrm{~S}$, $\mathrm{Ag}_{2} \mathrm{Se}$, and $\mathrm{Ag}$ Nanofibers by Using a General Sacrificial Template and Their Application in Electronic Device Fabrication. Advanced Functional Materials, 18(8), 1249-1256. doi: 10.1002/adfm.200700953

Wang, X., Um, I. C., Fang, D., Okamoto, A., Hsiao, B. S. y Chu, B. (2005). Formation of waterresistant hyaluronic acid nanofibers by blowingassisted electro-spinning and non-toxic post treatments. Polymer, 46(13), 4853-4867. doi: 10.1016/j.polymer.2005.03.058

Wang, Z. L. (2000). Characterizing the Structure and Properties of Individual Wire-Like Nanoentities. Advanced Materials, 12(17), 1295-1298. doi: 10.1002/1521-4095(200009)12:17<1295::AIDADMA1295>3.0.CO;2-B

Wen, P., Zong, M.-H., Linhardt, R. J., Feng, K. y Wu, H. (2017). Electrospinning: A novel nanoencapsulation approach for bioactive compounds. Trends in Food Science \& Technology, 70, 56-68. doi: 10.1016/j.tifs.2017.10.009

Wernsdorfer, W. y Sessoli, R. (1999). Quantum phase interference and parity effects in magnetic molecular clusters. Science, 284(5411), 133-135. doi: $10.1126 /$ science.284.5411.133

Xia, Y., Yang, P., Sun, Y., Wu, Y., Mayers, B., Gates, B., ... Yan, H. (2003). One-Dimensional Nanostructures: Synthesis, Characterization, and Applications. Advanced Materials, 15(5), 353-389. doi: 10.1002/ adma.200390087

Xu, C. Y., Inai, R., Kotaki, M. y Ramakrishna, S. (2004). Aligned biodegradable nanofibrous structure: a potential scaffold for blood vessel engineering. Biomaterials, 25(5), 877-886. Recuperado de http://www.ncbi.nlm.nih.gov/pubmed/14609676

Yang, F., Both, S. K., Yang, X., Walboomers, X. F. y Jansen, J. A. (2009). Development of an electrospun nanoapatite/PCL composite membrane for GTR/GBR 
application. Acta Biomaterialia, 5(9), 3295-3304.

doi: 10.1016/J.ACTBIO.2009.05.023

Yang, Y., Wen, J., Wei, J., Xiong, R., Shi, J. y Pan, C. (2013). Polypyrrole-Decorated $\mathrm{Ag}-\mathrm{TiO}_{2}$ Nanofibers Exhibiting Enhanced Photocatalytic Activity under Visible-Light Illumination. ACS Applied Materials \& Interfaces, 5(13), 6201-6207. doi: 10.1021/ am401167y

Yoo, H. S., Kim, T. G. y Park, T. G. (2009). Surfacefunctionalized electrospun nanofibers for tissue engineering and drug delivery. Advanced Drug Delivery Reviews, 61(12), 1033-1042. doi: 10.1016/J.ADDR.2009.07.007

Yuan, X., Zhang, Y., Dong, C. y Sheng, J. (2004). Morphology of ultrafine polysulfone fibers prepared by electrospinning. Polymer International, 53(11), 1704-1710. doi: 10.1002/pi.1538

Zheng-Ming, H., Zhang, Y.-Z., Kotaki, M. y Ramakrishna, S. (2003). A review on polymer nanofibers by electrospinning and their applications in nanocomposites. Composites Science and Technology, 63(15), 2223-2253. doi: 10.1016/ S0266-3538(03)00178-7 